\title{
A saúde do aluno com transtorno de déficit de atenção com hiperatividade em seu processo de ensino e aprendizagem: uma revisão bibliográfica
}

The health of students with attention deficit hyperactivity disorder in their teaching and learning process: a bibliographic review

La salud de los estudiantes con trastorno de déficit de atención e hiperactividad en su proceso de enseñanza y aprendizaje: una revisión bibliográfica

Patricia de Cerqueira Pretti ${ }^{1 *}$, Luana Frigulha Guisso

\section{RESUMO}

Objetivo: Objetiva-se nesta revisão exaltar a importância de conhecer de perto a realidade dos alunos com Transtorno de Déficit de Atenção com Hiperatividade (TDAH) e a necessidade de garantir um processo de ensino e aprendizagem não nocivos à sua saúde. Revisão Bibliográfica: O TDAH é uma das causas de dificuldade de aprendizado de natureza neurobiológica mais comum durante a infância e à adolescência, podendo acarretar sérios prejuízos no rendimento escolar e na capacidade de se apropriar da aprendizagem adequada. Os danos à saúde do aluno com TDAH que não recebe um tratamento adequado de inteiração e pouco debruçado em medidas paliativas, podem ser desastrosos, principalmente quando se considera as necessidades do aluno e não somente a ausência de preparo do ambiente escolar ao receber essa demanda. Entende-se, para tanto, que os valores e significados sobre o TDAH se refletem no modo como as pessoas lidam com a situação. Considerações finais: Na busca por respostas a esse questionamento de inclusão para uma aprendizagem assertiva, a revisão se pautou nas práticas atuais que podem comprometer a saúde do discente com TDAH e a necessidade de uma nova intervenção no campo interacionista.

Palavras-chave: TDAH, Saúde na escola, Saúde mental.

\begin{abstract}
Objective: The objective of this review is to highlight the importance of knowing the reality of students with Attention Deficit Hyperactivity Disorder (ADHD) and the need to ensure a teaching and learning process that is not harmful to their health. Bibliographic review: ADHD is one of the most common causes of learning disabilities of a neurobiological nature during childhood and adolescence, which can cause serious losses in school performance and in the ability to appropriate appropriate learning. The damage to the health of the student with ADHD who does not receive adequate treatment of interaction and little focused on palliative measures, can be disastrous, especially when considering the needs of the student and not only the lack of preparation of the school environment when receiving this demand. Therefore, it is understood that the values and meanings about ADHD are reflected in the way people deal with the situation. Final considerations: In the search for answers to this question of inclusion for assertive learning, the review was based on current practices that can compromise the health of students with ADHD and the need for a new intervention in the interactionist field.
\end{abstract}

Key words: ADHD, Health at school, Mental health.

${ }^{1}$ Faculdade Vale do Cricaré (FVC). São Mateus - ES. *E-mail: pcpretti@gmail.com 


\section{RESUMEN}

Objetivo: El objetivo de esta revisión es resaltar la importancia de conocer de cerca la realidad de los estudiantes con trastorno por déficit de atención con hiperactividad (TDAH) y la necesidad de garantizar un proceso de enseñanza y aprendizaje que no sea perjudicial para su salud. Revisión bibliográfica: el TDAH es una de las causas más comunes de dificultades de aprendizaje de naturaleza neurobiológica durante la infancia y la adolescencia, que puede causar serias pérdidas en el rendimiento escolar y en la capacidad de apropiarse del aprendizaje apropiado. El daño a la salud del estudiante con TDAH que no recibe un tratamiento adecuado de la interacción y está poco enfocado en las medidas paliativas, puede ser desastroso, especialmente cuando se consideran las necesidades del estudiante y no solo la falta de preparación del entorno escolar al recibir esta demanda. Por lo tanto, se entiende que los valores y significados sobre el TDAH se reflejan en la forma en que las personas manejan la situación. Consideraciones finales: en la búsqueda de respuestas a esta pregunta de inclusión para el aprendizaje asertivo, la revisión se basó en prácticas actuales que pueden comprometer la salud de los estudiantes con TDAH y la necesidad de una nueva intervención en el campo interaccionista.

Palabras clave: TDAH, Salud en la escuela, Salud mental.

\section{INTRODUÇÃO}

O Transtorno de Déficit de Atenção com Hiperatividade (TDAH) é uma das causas de dificuldade de aprendizado de natureza neurobiológica mais comum durante a infância e à adolescência, podendo acarretar sérios prejuízos no rendimento escolar e na capacidade de se apropriar da aprendizagem adequada com lacunas de conteúdos e potencialização ou desenvolvimento de outros distúrbios. O TDAH continua sendo um problema clínico significativo com que se defrontam famílias, médicos, professores, pedagogos, psicólogos e educadores. É um distúrbio crônico, ou seja, os sintomas existem continuamente, e com duração de no mínimo seis meses (MATTOS P, 2003).

Ressalta-se neste contexto, como estratégia fundamental, a adoção de formas e meios pedagógicos para otimizar e melhorar o engajamento atencional da criança com TDAH. A escola precisa participar do processo terapêutico formulando práticas e caminhos que facilite e otimize a absorção de conteúdos e a desenvoltura nas avaliações a partir da teoria de aprendizagem adotada pela escola, baseada no comportamento, do aspecto humano ou, apenas a capacidade cognitiva de cada um, mas que precisa estar afinada com as necessidades do educando (CRUZ MGA, et al., 2016) .

Vale destacar que não se trata apenas de um estado temporário; não é causado por falta de disciplina ou controle parental. Na prática, o TDAH deve ser conduzido como um estudo da neurociência, que é uma ciência interdisciplinar que colabora com outros campos como a educação, química, ciência da computação, engenharia, antropologia, linguística, matemática, medicina e disciplinas afins, filosofia, física, comunicação e psicologia. O TDAH é considerado uma perturbação do desenvolvimento da infância e adolescência mais estudado e o distúrbio neurocomportamental mais frequente na criança de idade escolar (SENO MP, 2010).

As inúmeras comorbidades podem ser detectadas em associação ao Transtorno de Déficit de Atenção/Hiperatividade, como: problemas de conduta (transtorno desafiador de oposição, que é um problema caracterizado por atitudes antissociais apresentadas geralmente por crianças e adolescentes; implicações emocionais (baixa autoestima ou depressão); problemas de socialização; problemas familiares; dificuldades no desempenho acadêmico e habilidades cognitivas e linguísticas (dificuldades na realização de problemas complexos ou problemas de fala e linguagem) e dificuldades com a saúde (EFFGEM V, et al., 2017).

A psicopedagogia institucional-escolar procura refletir sobre a aprendizagem do aluno, com a extrema relação com a informação e os conceitos em diferentes áreas do conhecimento, buscando os diagnósticos e ampliação da prática em sala de aula, junto com os professores e pedagogos. A psicopedagogia é um campo de atuação, que podemos identificar a princípio, a junção da pedagogia com a psicologia, com o objetivo do desenvolvimento de estratégias para serem trabalhadas com crianças com dificuldades 
de aprendizagem, seja ela, na parte escolar, social e comportamental, onde há de se observar com atenção todas as dificuldades de aprendizagem, valorizando o processo de desenvolvimento do aluno/paciente, em suas diversas dimensões, tais como as afetivas, cognitivas, orgânica e psicossocial (SIGNOR RCF, et al., 2016).

A obtenção do diagnóstico pode ocorrer de várias maneiras, porém, a entrada da criança no ambiente escolar se constitui em um marco decisivo que evidencia o aparecimento do transtorno. O transtorno de atenção se configura como uma patologia da criança que precisa ser tratada, no intuito de promover alguma melhora no desempenho escolar ou em seu comportamento.

O uso de medicamentos pode-se fazer necessário, lembrando que, embora nem todos precisem da medicação, ela é eficiente nos casos em que os sintomas impedem ou atrapalham a intervenção psicoterápica e psicopedagógica, pois ela exerce controle dos principais sintomas e dos prejuízos provocados pela doença. É importante ressaltar que quando a intervenção é feita precocemente, pode-se minimizar o impacto negativo causado pela dificuldade (SIGNOR RCF, et al., 2016).

Objetiva-se, portanto, no desenvolvimento dessa pesquisa, reunir a literatura disponível e apontar as possíveis intromissões da medicalização irresponsável em casos que, o tratamento através da inteiração do aluno surte um efeito mais desejado e colabora com o desenvolvimento da criança, promovendo superação e construção da quebra de paradigmas. A medicalização pode ser o caminho mais fácil, mas não está classificada como único caminho, já que por inúmeras vezes o interesse do paciente de TDAH não é levado em conta. A escolha do tema desta pesquisa foi motivada por se considerar importante conhecer de perto como acontece o processo de ensino e aprendizagem e as implicações na saúde do aluno com déficit atencional com o emprego das práticas atuais.

\section{REVISÃO BIBLIOGRÁFICA}

O transtorno do déficit de atenção, mais conhecido como Distúrbio de Déficit de Atenção (DDA), em sua etiologia, é uma condição em que há falta de atenção, hiperatividade, impulsividade e agitação. As características do TDAH aparecem na infância e o comportamento é crônico, com duração de no mínimo seis meses. É visto como um transtorno orgânico, de ordem neurológica, que compromete a aprendizagem e prejudica o desempenho escolar, além do familiar, emocional, social e laboral ao longo da vida, causando sofrimento nas crianças atingidas e suas famílias.

Vale destacar que não se trata apenas de um estado temporário; não é causado por falta de disciplina ou controle parental e nem todos os indivíduos têm o mesmo grau de comprometimento das funções (LUIZÃO AM e SCICCHITANO RMJ, 2014). O modelo comportamental define o transtorno partindo da avaliação do comportamento no ambiente, estudando as situações específicas nas quais eles surgem. Eles apontam para a existência de um padrão de condutas concretas, manifestadas em respostas as condições ambientais adversas.

O enfoque psicanalítico, compreende que este transtorno é a forma de evadir a fantasia de morte ou funciona também como mecanismo de compensação de estados de ansiedade motivados por depressão. os principais parâmetros para realização de um diagnóstico preciso do TDAH são os sintomas clínicos, sendo estes a: desatenção, a hiperatividade e a impulsividade que a criança ou o adolescente apresentam no percurso de seu desenvolvimento comprometendo suas rotinas e suas relações pessoais e interpessoais na escola, no trabalho e em casa (LARROCA LM e DOMINGOS NC, 2012).

A Lei Brasileira de Inclusão atribui ao poder público o dever de garantir condições de acesso, permanência, participação e aprendizagem das pessoas com deficiência, em uma determinação clara de que o acesso não é suficiente, mas que deve haver condições de permanência, participação e aprendizagem, para que a inclusão aconteça verdadeiramente. As políticas de inclusão dependem das condições do discente de participar do processo educacional de maneira ativa, o que pode ser prejudicado quando existe a inserção indesejada do medicamento (FREITAS MC e JACOB RNF, 2019). 
"A lei dispõe que: Art. 28. Incumbe ao poder público assegurar, criar, desenvolver, implementar, incentivar, acompanhar e avaliar: I - Sistema educacional inclusivo em todos os níveis e modalidades, bem como o aprendizado ao longo de toda a vida; II Aprimoramento dos sistemas educacionais, visando a garantir condições de acesso, permanência, participação e aprendizagem, por meio da oferta de serviços e de recursos de acessibilidade que eliminem as barreiras e promovam a inclusão plena; III - projeto pedagógico que institucionalize o atendimento educacional especializado, assim como os demais serviços e adaptações razoáveis, para atender às características dos estudantes com deficiência e garantir o seu pleno acesso ao currículo em condições de igualdade, promovendo a conquista e o exercício de sua autonomia [...]" (BRASIL, 2015).

É oportuno lembrar que, ao longo do tempo, o TDAH recebeu diversas denominações, como por exemplo, lesão cerebral mínima ou disfunção cerebral mínima (dificuldade em controlar impulsos, manter a atenção, e realizar atividades motoras que exijam coordenação e equilíbrio) e síndrome hipercinética (de conduta desnecessária, desorganizada e caótica, caracterizada pela combinação de inquietude e falta de atenção em um nível impróprio para a idade da criança). A versão atualizada do Diagnostic and Statistical Manual (DSM) saiu em maio de 2013 e substitui o DSM-IV criado em 1994 e revisado em 2000. No DSM, o TDAH agora ocorre durante toda a vida, foram criados os subtipos de TDAH e possível diagnóstico de TDAH junto com autismo, além disso, deixou de ser um problema de desenvolvimento para ser um problema neurológico (DSM-IV, 2015).

A teoria encoraja e valida o modelo educativo de competência, pois entende-se a escola como sistema organizado que precisa respeitar as diferentes formas e ritmos de aprendizagem, opondo-se ao modelo em que as incapacidades e competências cognitivas das pessoas com TDAH são evidenciadas pura e simplesmente. A Teoria Histórico-Cultural de Vygotsky, em sua gênese, pressupõe uma natureza social da aprendizagem, ou seja, é por meio das interações sociais que o indivíduo desenvolve suas funções psicológicas superiores.

A preocupação central de Vygotsky refere-se ao desenvolvimento das funções psicológicas superiores, que distinguem o ser humano dos demais animais: atenção e memória voluntária, memorização ativa, pensamento abstrato, raciocínio dedutivo e capacidade de planejamento, além da linguagem que, para Vygotsky, era a principal função psicológica superior. Também conhecida como abordagem sociointeracionista, toma como ponto de partida as funções psicológicas dos indivíduos, as quais classificou de elementares e superiores, para explicar o objeto de estudo da sua psicologia: a consciência (VYGOTSKY LS, 2007).

Em uma sociedade hiperativa, onde as crianças estão submetidas a inúmeros estímulos, o professor e aluno são considerados sujeitos do processo ensino-aprendizagem, cabendo aos educadores pensar 0 trabalho pedagógico com essa diversidade também. Os alunos considerados problema nas salas de aula, podem ser aqueles portadores do TDAH, os distraídos ou simplesmente uma criança sem limites - muito comum na sociedade atual. Identificar e tratar, não significa apenas promover métodos comuns do cotidiano. O desenvolvimento de pesquisa e novas práticas são consideradas intervenções de tratamento, e disponibilizam alternativas promissoras (DOMINGUES L, et al., 2013).

\section{A teoria Sociointeracionista de Vygotsky como alternativa}

A teoria Sociointeracionista de Lev Semyonovich Vygotsky, defende que o contexto histórico-cultural exerce papel importante no desenvolvimento do ser, ou seja, o desenvolvimento se dá através das relações que se tem com o meio que vive e do convívio que com outros seres humanos. À partir daí que nasce a construção do conhecimento e das individualidades, existe uma construção de saberes à partir da reunião de toda influência recebida (ZANOLLA SRS, 2012).

Embora cada um possua características únicas, inclusive na maneira de aprender, acredita-se que a investigação no ambiente escolar por pesquisadores da área da educação possa contribuir significativamente. 
Ao aluno, a informação precisa ser organizada para ser interiorizada, para tornar seu o conhecimento, o seu saber. O conhecimento tem de ser um bem comum e a aprendizagem um direito de todos. Nesse movimento, segundo a teoria de Vygotsky, a escola não passa ilesa e sim ganha contornos diferenciados de acordo com a organização do trabalho, representando as necessidades de cada época, expressas e vivenciadas por homens reais, aqueles que existem de fato, na realidade, opondo-se ao que não existe. Ele amplia a importância da aprendizagem escolar, da escola e do professor no desenvolvimento das funções psicológicas superiores, reafirmando que a atenção e o controle voluntário do comportamento são mediados pelas interrelações estabelecidas entre o adulto e a criança (EIDT NM e TULESKI SC, 2010).

A escola conteudista frequentada pelo público alvo, transita de forma confusa em sua teoria de aprendizagem, ora com abordagem a partir do aspecto humano, ora apenas avaliando a capacidade cognitiva de cada um, levando alguns alunos a terem dificuldades para fazerem as atividades de casa sozinhos. Os que se distraem a todo instante, precisam ser sempre monitorados em casa ou contar com apoio de aulas de reforço fora do ambiente escolar. Na perspectiva de Vygotsky, o aprendizado deve caminhar à frente do desenvolvimento. A relação entre função executiva e aprendizagem escolar mediadas pelo professor, apontam o controle atencional como um fator determinante no desempenho das crianças (GOMES RC, et al., 2010).

Considerando que na infância o cérebro é mais maleável e que a plasticidade cerebral é mais comum em crianças através das janelas de oportunidades, temos nas práticas pedagógicas, em especial no ensinoaprendizagem de crianças com TDAH, o cenário para a linha dessa pesquisa: o ambiente escolar, porque não somos apenas um cérebro que processa as informações percebidas e um corpo que atua com base nestes registros, somos seres afetados pelo ambiente com suas dificuldades e potencialidades. As obras de Vygotsky na área da psicologia da educação significaram novas possibilidades para se pensar o processo de aprendizagem, de desenvolvimento cognitivo e mesmo a importância da intervenção pedagógica (PINO A, 2010).

Otimizar as janelas de oportunidades é um investimento pautado num somatório de ações que vão desde o ambiente social a ação educativa. Estímulos bem direcionados podem mudar uma predisposição genética. Contudo, devemos considerar o contexto da ação, pois esses estímulos precisam acontecer de forma consistente e consciente de seus benefícios, tanto no ambiente escolar como no familiar. As informações acerca dos potenciais a serem desenvolvidos nas crianças precisam estar afinadas nos seus ambientes, a fim de não delegarmos apenas a medicina a tarefa de normatizar e legislar sobre a vida humana. Precisamos respeitar os estímulos naturais próprios e oportunos na infância e o espaço escolar torna-se elemento fundamental para esta investigação. Sendo assim, as entidades escola, saúde e família, as mais eloquentes da vivência humana, precisam estar em sintonia nas suas relações de poder (ASSEMANY NM, 2016).

Entende-se, portanto, que o professor mediador pode interceder com as estratégias de aprendizagem, elenco de funções superiores (objeto da Teoria Histórico-Cultural de Vygotsky): atenção, percepção, memória, linguagem, metacognição - o indivíduo regula seu pensamento e ação.

A essas funções superiores chamamos de Função Executiva, um conjunto de práticas voltadas para o aspecto cognitivo, cujo objetivo é o de colaborar com diversas tarefas que necessitam de itens importantes, a saber: planejamento e monitoramento de comportamentos cuja intenção é relacionada a um determinado objetivo ou então, a demandas de caráter ambiental (NAVARRO MS e PRODÓCIMO E, 2012).

\section{A saúde comprometida pela intervenção da medicalização indesejada}

O TDAH foi constituído na economia biomédica da atenção, característica das últimas décadas do século $X X$. Deste modo, todo problema que envolve a educação se torna de cunho biológico em que é somente da criança o problema. Pouco se questiona sobre o processo de ensino-aprendizagem, nem tampouco sobre a formação despendida aos docentes, que hoje se veem constrangidos por uma atmosfera cultural pouco reflexiva que prioriza a circulação e o consumo de materiais pedagógicos padronizados pelo mercado, recentemente ofertados mediante o uso das Tecnologias da Informação e Comunicação - TICs, em detrimento dos processos formativos presenciais (BRZOZOWSKI FS e CAPONI S, 2009). 
Ross A (2016) destaca que, a alteração do comportamento é mais rápida e mais fácil com o uso da droga, o que faz o terapeuta recorrer ao medicamento como principal plano de tratamento aos quadros de TDAH e problemas de aprendizagem. Todavia, alerta o autor, nessa dinâmica, ocorre o aumento potencial do uso do medicamento que, segundo ele, está atingindo um patamar de irresponsabilidade. As afirmações do autor datam da década de 1979, mas se mostram atuais e ainda mais pertinentes em nossos dias, em razão do recorrente diagnóstico de TDAH e do uso abusivo do metilfenidato por crianças, adolescentes e jovens.

Uma intervenção para ser eficiente deve buscar o que leva ao surgimento do sintoma e quais condições favorecem seu surgimento. Da mesma forma, dar excessiva ênfase às influências externas de modo a direcionar o tratamento a ela, pode levar ao erro de se esquecer que o sujeito apresenta dificuldades por uma tendência própria a desenvolvê-las. Quando a criança ou adolescente estiver no processo de avaliação diagnóstica ou mesmo já fazendo o tratamento interventivo, o profissional pode focalizar dificuldades específicas da criança em termos de habilidades sociais, criando um espaço e situações para desenvolvêlas, por meio da interação com a criança por intermédio de qualquer atividade lúdica (CRUZ BA, et al., 2016).

A ludoterapia é uma técnica psicoterápica de abordagem infantil que se baseia no fato de que brincar é um meio natural de auto expressão da criança. O brincar terapêutico tem sido objeto de estudo nas diferentes linhas teóricas da Psicologia tais como a Psicanálise, Cognitivo Comportamental e o Humanismo, pois é considerada como mais uma possibilidade diagnóstica e/ou terapêutica nos atendimentos. Neste contexto de intervenção não farmacológica apenas, foram pesquisadas algumas práticas associadas a mudanças no estilo de vida, que podem ser consideradas importantes para o controle do TDAH. Dentre elas, as práticas de atividades físicas, a acupuntura, a yoga, Mindfulness, neurofeedback e os jogos (CRUZ BA, et al., 2016).

Considera-se que compreender melhor os benefícios da alimentação e da prática de atividades físicas podem ter a manifestação dos sintomas do TDAH reduzidos, uma vez que o exercício físico melhora a produção de dopamina assim como de outros neurotransmissores, tais como serotonina e endorfina. A liberação desses neurotransmissores estimula o funcionamento cognitivo e comportamental da pessoa. A acupuntura preconiza que a saúde depende das funções psico-neuroendócrinas, sob influência do código genético e de fatores extrínsecos como a nutrição, os hábitos de vida, o clima, a qualidade do ambiente, e todos os outros elementos da natureza que, de alguma forma, exercem pressão (positiva ou negativa) sobre a vida humana (TOPCZEWSKI A, 2014).

A revolução pessoal de crescimento inspirada em seu próprio raciocínio é o que os psicólogos chamam de experiência de descoberta que é motivada por um ambiente que reconhece nas relações a importância transformadora do esforço e de que maneira ele modifica o impacto do fracasso na escola, no esporte, no trabalho e nos relacionamentos e onde os diferentes fatores: estruturação do ambiente; nível de motivação; saliência da tarefa e estágio de desenvolvimento fazem todo o sentido em cada aluno/paciente que pode manifestar os sintomas do TDAH de forma diferente conforme sua idade e grau de desenvolvimento, bem como de acordo com suas habilidades e capacidades cognitivas. Finalmente, a conquista do equilíbrio está em possibilitar-Ihe uma ação integradora das potencialidades e do que a criança já conquistou para que possa construir ou resgatar o que lhe falta, não na ênfase em suprir o que falta à criança com dificuldades (TOPCZEWSKI A, 2014).

\section{CONSIDERAÇÕES FINAIS}

Tendo em vista os resultados desta revisão, concluímos que a comunidade escolar convive com a criança com TDAH, mas não se sente parte direta do seu processo de desenvolvimento. Há falta de informação e receio dos profissionais e da escola quanto aos sintomas da patologia. Verificamos a preferência da comunidade escolar para o uso da medicação e poucas citações acerca de outros tratamentos alternativos. Vale ressaltar que o desenvolvimento humano é resultado de interações dinâmicas e bidirecionais entre o indivíduo ativo e o ambiente físico e social, incluindo a família, escola e os centros de saúde. Atribuir a medicação a responsabilidade da ação na dificuldade de atenção ou de comportamento, fará com que percamos o foco nas políticas educacionais, na formação dos professores, na qualidade de ensino das escolas e do papel dos pais, deixando a criança como responsável pelo próprio fracasso escolar. 


\section{REFERÊNCIAS}

1. ASSEMANY NM. Superestimulação na infância: uma questão contemporânea. Cadernos de psicanálise (Rio de Janeiro), 2016; 38(34): 231-243.

2. BRASIL. 2015. In: LEI № 13.146, DE 6 DE JULHO DE 2015, Lei Brasileira de Inclusão da Pessoa com Deficiência. Disponível em: http://www.planalto.gov.br/ccivil_03/_ato2015-2018/2015/lei/l13146.htm. Acesso em: 12 jan. 2020.

3. BRZOZOWSKI FS e CAPONI S. Transtorno de Déficitde Atenção com Hiperatividade: classificação e classificados. Revista de Saúde Coletiva, 2009; 19(4): 1165-1187.

4. CRUZ BA, et al. Uma crítica à produção do TDAH e a administração de drogas para crianças. Estudos de Psicologia (Natal), 2016; 21(3): 282-292.

5. CRUZ MGA, et al. O caso Transtorno do Déficit de Atenção e Hiperatividade (TDAH) e a medicalização da educação: uma análise a partir do relato de pais e professores. Interface (Comunicação, saúde, educação), 2016; 20(58): 703714.

6. DOMINGUES L, et al. Transtorno de déficit de atenção/hiperatividade: um olhar voltado para a escola. Barbaroi, 2013; 39(20): 149-163.

7. DSM-IV. Manual Diagnóstico e Estatístico de Transtornos Mentais. 3nd ed. Porto Alegre: Artes Médicas, 2015; 8990p.

8. EFFGEM V, et al. A visão de profissionais de saúde acerca do TDAH - processo diagnóstico e práticas de tratamento. Construção psicopedagógica, 2017; 25(26): 34-45.

9. EIDT NM, TULESKI SC. Transtorno de Déficit de Atenção/Hiperatividade e psicologia Histórico-Cultural. Cadernos de Pesquisa, 2010; 40(139): 121-146.

10. FREITAS MC, JACOB RNF. Inclusão educacional de crianças com deficiências: notas do chão da escola. Educação e Pesquisa, 2019; 45(1): 1-10.

11. GOMES RC, et al. Teorias de aprendizagem: pré-concepções de alunos da área de exatas do ensino superior privado da cidade de São Paulo. Ciência \& Educação (Bauru), 2010; 16(3): 695-708.

12. LARROCA LM, DOMINGOS NM. TDAH - Investigação dos critérios para diagnóstico do subtipo predominantemente desatento. Psicologia Escolar e Educacional, 2012; 16(1): 113-123.

13. LUIZÃO AM, SCICCHITANO RMJ. Transtorno de déficit de atenção e hiperatividade: um recorte da produção científica recente. Revista Psicopedagogia, 2014; 31(96): 289-297.

14. MATTOS P. No mundo da lua: perguntas e respostas sobre transtorno do déficit de atenção com hiperatividade em crianças, adolescentes e adultos. 2nd ed. São Paulo: Lemos Editorial, 2003; 110p.

15. NAVARRO MS e PRODÓCIMO E. Brincar e mediação na escola. Revista Brasileira de Ciências do Esporte, 2012; 34(3): 633-648.

16. PINO A. A criança e seu meio: contribuição de Vigotski ao desenvolvimento da criança e à sua educação. Psicologia USP, 2010; 21(4): 741-756.

17. ROSS A. Dificuldades do processo de aprendizagem. 5nd ed. Rio de Janeiro: Wak editora, 2016; 116p.

18. SENO MP. Transtorno do déficit de atenção e hiperatividade (TDAH): o que os educadores sabem?. Revista Psicopedagogia, 2010; 27(84): 334-343.

19. SIGNOR RCF, et al. A medicalização da educação: implicações para a constituição do sujeito/aprendiz. Educação e Pesquisa, 2016; 43(3): 743-763.

20. TOPCZEWSKI A. Transtorno do déficit de atenção e hiperatividade: uma vertente terapêutica. Einstein, 2014; 12(3): 310-313.

21. VYGOTSKY LS. Psicologia Pedagógica para a teoria histórico-cultural de Vigotski. 1ed. São Paulo: Scipione, 2007; $201 \mathrm{p}$.

22. ZANOLLA SRS. O conceito de mediação em Vigotski e Adorno. Psicologia \& Sociedade, 2012; 24(1): 5-14. 